\title{
The Determination of the Total Efficiency for NaI(Tl) Detector by GATE Simulation
}

\author{
Nuray YAVUZKANAT ${ }^{1 *}$, Didem GÜNGÜR ${ }^{2}$, Sezai YALÇIN ${ }^{1}$ \\ ${ }^{1}$ Bitlis Eren University, Department of Physics, Bitlis \\ ${ }^{2}$ Bitlis Eren University, Graduate School of Natural and Applied Sciences, Bitlis \\ (ORCID: 0000-0001-5055-9185) (ORCID: 0000-0003-2742-1094) (ORCID: 0000-0001-6779-9230)
}

\begin{abstract}
In this study the total efficiency of the $\mathrm{NaI}(\mathrm{Tl})$ detector have been calculated by using Geant 4 based GATE simulation program. The simulation was performed using point and disc isotropic gamma-ray sources at various distance between source and detector in the photon energy range 50-3000 keV. Results were compared with different analytical and Monte Carlo calculations obtained in the time of the periods 1958-2018. The calculated and simulated data indicated good agreement with finding more sensitive GATE simulation result in the lower energy range from $50 \mathrm{keV}$ to $150 \mathrm{keV}$.
\end{abstract}

Keywords: NaI(Tl), Total Efficiency, Geant4, GATE.

\section{NaI(TI) Dedektörünün GATE Simülasyon Programıla Toplam Veriminin Belirlenmesi}

\begin{abstract}
$\ddot{O} \mathbf{z}$
$\mathrm{Bu}$ çalışmada Geant4 tabanlı GATE simulasyon programı kullanılarak NaI(Tl) detektörünün toplam verimi hesaplanmıştır. Simulasyon, 50-3000 keV foton enerji aralığında çeşitli kaynak-detektör uzaklıklarında izotropik nokta ve disk gama ışını kaynağı kullanılarak gerçekleştirilmiştir. Sonuçlar, 1958-2018 periyodu boyunca elde edilen farklı analitik ve Monte Carlo hesaplamaları sonuçları ile karşılaştırılmıştır. Hesaplanan ve GATE simulasyonuyla elde edilen sonuçların birbiriyle uyumlu çıkmasının yanında, bu çalışmada $50 \mathrm{keV}$ ile $150 \mathrm{keV}$ arasındaki düşük enerjideki gamalar için çok daha hassas sonuçlar bulunmuştur.
\end{abstract}

Anahtar kelimeler: NaI(Tl), Toplam Verim, Geant4, GATE.

\section{Introduction}

Scintillation detectors are the main class of radiation detectors used in many areas (eg in high energy physics, nuclear physics, nuclear medicine, industry, security, geology, astrophysics, agriculture, radiation measurement of environmental samples, etc.) [1-4]. Hofstadter in 1948 firstly showed that thallium-activated sodium iodide crystals (NaI(Tl)) combining with PMTs (Photomultiplier Tubes) used as an efficient detector to measure gamma-rays and other ionizing radiations [5]. Therefore, $\mathrm{NaI}(\mathrm{Tl})$ is the first solid-state detector used as a gamma-ray spectrometer in the 50s and after that time it is still the most common gamma-ray detector system [6]. $\mathrm{NaI}(\mathrm{Tl})$ is a highly popular detector due to its good efficiency, wide operating temperature range, low consumption and low costs, as well as in-suit radioactivity measurements $[7,8]$. Today it is the most important part of nuclear medicine (used in mammography, gamma cameras, and positron emission tomography) as well as astrophysics. The NaI (T1) detectors are also used in the measurement of the environmental radioactivity in the marine as used in the Fukushima nuclear accident [9].

*Sorumlu yazar: nyavuzkanat@beu.edu.tr

Geliş Tarihi: 20.11.2019, Kabul Tarihi:19.12.2019 
The most important properties of the radiation detector are the detector response function, the total efficiency (TE), the full energy peak efficiency (FEPE) and the detector resolution. It is necessary to determine the detector properties to make gamma- ray measurements over a wide energy range. In the gamma-ray activity measurement, there are two critical properties in detector efficiency; total efficiency and full energy peak energy efficiency. To measure the absolute activities from the gammaray source over a wide energy range, the total efficiency needs to be known. The total efficiency is described by four different methods as experimental, empirical, analytical and Monte Carlo approach [10]. It is necessary to use standard sources to determine the total efficiency in the experimental method. These standard sources are quite expensive and require time to prepare in the laboratory [11]. It is also difficult to find standard gamma sources in the desirable energy range. Instead of the determination of the total detector efficiency by the experimental method, it is easy to fit the measurement values by analytical function or use the computer program based on the gamma-rays interactions with the detector [12]. One of the important analytical methods calculates the efficiency via identifying the average chord length which is called Mean Chord Length (MLC) $[13,14]$. In addition to experimental and computerbased calculations, new published analytical studies are directly calculated the detector efficiency for various detector-source arrangements [10,15-17].

Monte Carlo (MC) simulation method, used in many fields from the medical physics to particle physics and keeps its popularity. Nowadays it is widely used to calculate the application parameters such as the activity measurement, the detector efficiency, coincidence summing corrective factor in gamma-ray spectroscopy. Well-known multi-purpose MC codes are PENELOPE, MCNP, Geant4, GATE, FLUKA, EGS, etc. [18].

GATE is an advanced open- source software developed by international collaborations and dedicated to the medical applications and radiotherapy. Recently it plays a key role in terms of designing new medical devices, acquisition protocols, quality control systems of the medical imaging devices and image reconstructions. It is also used in the characterization of the detector system which is the most important part of the positron emission tomography (PET), etc. GATE runs with Geant 4 simulation toolkit which is the validated physics models, complex geometrical volume description and powerful 3D visualization (such as Qt mode) [8, 19-23].

In this study, the total efficiency of the $\mathrm{NaI}(\mathrm{Tl})$ detector was obtained using the Geant 4 based GATE simulation program. The simulation code was modeled for 3" 3 " and 2"x2" cylindrical NaI(Tl) detector systems and obtained the output as a root file for every gamma-ray energy from $50 \mathrm{keV}$ to 3000 $\mathrm{keV}$. A simulated energy spectrum was used to calculate the total efficiency of the detector and compared the result with published results by using analytical and MC calculations. Founded the total efficiency values with GATE simulation were found to agree with the literature.

\section{Material and Method}

\section{Modeled GATE (Geant4 Application for Tomographic Emission) Simulation}

We simulated the photon detection efficiency of the $\mathrm{NaI}(\mathrm{Tl})$ detector with Geant 4 based GATE simulation program. The simulation was performed using the different shapes of the sources at various distances (between source and detector) in the photon energy range 50-3000 keV. The versions of the GATE and Geant 4 were 8.0 and 10.3 respectively. Firstly, a cylindrical $\mathrm{NaI}(\mathrm{Tl})$ detector was defined as a three-inch length and three-inch diameter. The point source was positioned at $0.001,0.5$ and $10 \mathrm{~cm}$ distance from the detector surface. The disc source was also determined in the simulation with a radius of $3.18 \mathrm{~cm}$ and located at 3 and $10 \mathrm{~cm}$ away from the detector surface. Moreover, 2"x2" $\mathrm{NaI}(\mathrm{Tl})$ detector was also defined in the simulation and calculated the total efficiencies of the detector for $0.001,5$ and $10 \mathrm{~cm}$ detector-source distance to compare the result with the current publication. Simulation setup and disc source visualization in Geant 4 is shown in Figure 1. 


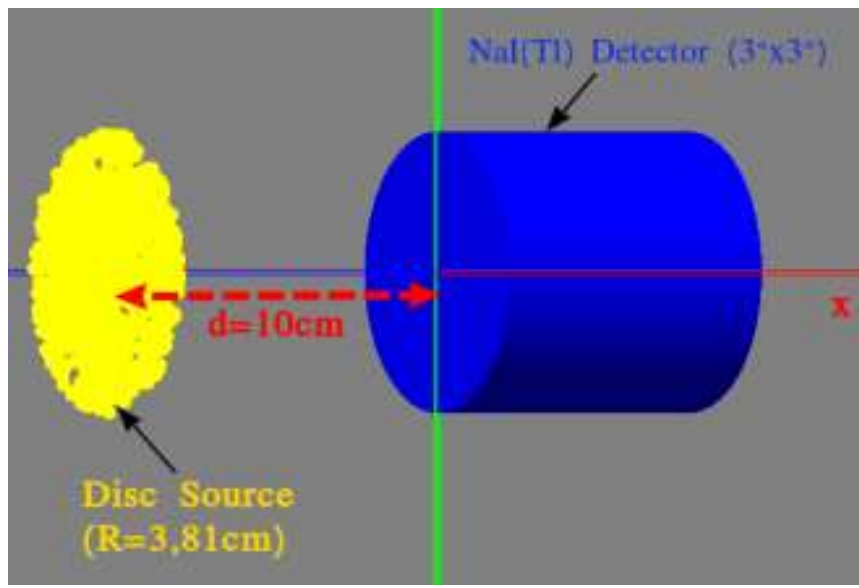

Figure 1. Geant4 visualization of the simulation setup for detecting gamma-rays emitting from disc source. Blue cylinder volume was defined for $\mathrm{NaI}(\mathrm{Tl})$ scintillation material with the size of 3"x3". Yellow points represent the disc source with radius of $\mathrm{R}=3.81 \mathrm{~cm}$

In Figure 2, gamma-rays irradiated from the disc source located at $10 \mathrm{~cm}$ distance from the detector. Short red lines represent the travel distance of the electron that occurs after the interaction between scintillation and gamma-rays. Green long lines classically represent the photons in the Geant 4 .

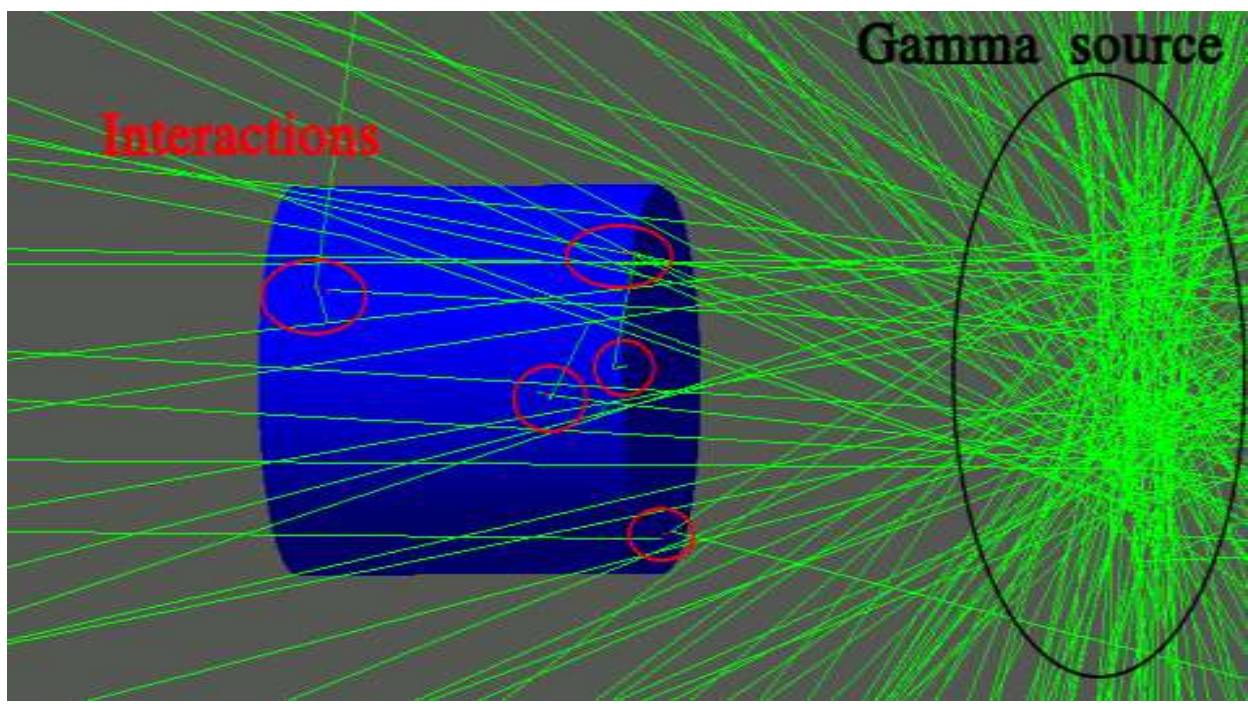

Figure 2. Green lines which is coming from the disc source (showing with black circle in the fig.) represent gamma rays in Geant4 simulation. Red circles in the figure show the interactions between scintillation and gamma-rays. Red lines indicate the electrons in the Geant4 simulation. In the simulation, detector volume was also capsuled with thin aluminum layer

Secondly, the physics list was chosen according to the photons energy range, therefore the physics-list builder name was called emstandard (electromagnetic physics-list) provided by the Geant 4 . After defining the system, source, digitization, and readout parameters, lastly, we described the data output as a root file that give all functionalities to achieve big data processing, numerical analysis and storage [30]. All occurred data outputs in the simulation were analyzed by ROOT software. The energy spectrum was obtained from the GATE simulation by recording all the interactions and the deposit energy of the detector crystal. Total efficiencies were calculated using the following equation:

TotalEfficiency $=\frac{N_{D}}{N}$ 
where $\mathrm{N}_{\mathrm{D}}$ is the number of the detected photons by the scintillation, and $\mathrm{N}$ represents the number of photons emitted by the source. The net count of the emitted photons was stabilized with one million gamma-rays for each different case in the simulation. Detected photon number was found from the energy spectrum and all results were listed in the Table 1 and Table 2.

\section{Result and Discussion}

The total gamma efficiencies of the $\mathrm{NaI}(\mathrm{Tl})$ detector for the disk and point sources were evaluated at a different detector-source distances in the various energy ranges from $50 \mathrm{keV}$ to $3000 \mathrm{keV}$. The total photon efficiencies for the disk and point sources obtained from the GATE simulation were compared with the calculated efficiencies by various analytical Monte Carlo approaches over the time period (between 1958 and 2018). In Table 1, there is a comparison between simulation with GATE and analytical calculations for $\mathrm{NaI}(\mathrm{Tl})$ detector's photon efficiency. In that part of the simulation, the disc source radius was defined as a $3.81 \mathrm{~cm}$ and located at two different positions $(3$ and $10 \mathrm{~cm}$ away from the detector surface). The analytical calculations and simulation results showed in good agreement. Especially, present work results consistent with the hybrid Monte Carlo method results calculated by [3]. Figure 3 shows that comparison between this work with different analytical methods and Monte Carlo techniques in terms of the total photon efficiencies for $\mathrm{NaI}(\mathrm{Tl})$ scintillator in the energy range between $50 \mathrm{keV}$ and $3000 \mathrm{keV}$. In the Figure 3 (b) there is a slight discrepancy over the $1332 \mathrm{keV}$ between this work and others (exception of the [3] and [27] works).

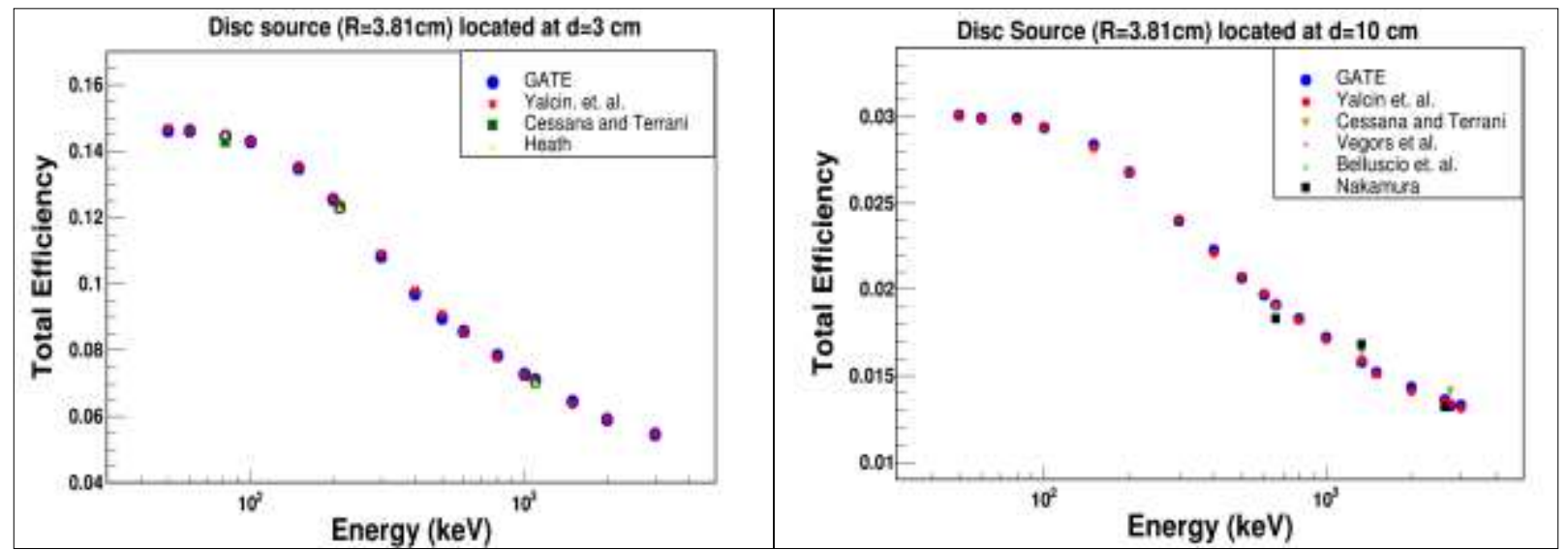

Figure 3. Total photon efficiency for a 3"x3" NaI(Tl) scintillator with a $3.18 \mathrm{~cm}$ radius disc source positioned at (a) $3 \mathrm{~cm}$ and (b) $10 \mathrm{~cm}$ distance between source and the detector surface

In Table 2, Geant 4 based GATE simulation result for point source is compared with an analytical and different types of Monte Carlo calculations in total photon efficiency of the $\mathrm{NaI}(\mathrm{Tl})$ detector. The point source was positioned at three different places with $0.001,0.5$ and $10 \mathrm{~cm}$ away from the detector. The analytical calculation (except Mean Chord Length) and different types of Monte Carlo calculations consistent with GATE simulation results. Figure 4 also indicates that the comparison between this study and different analytical methods or Monte Carlo techniques. In the Figure 4 (a), (b) and (c), the divergence between MCL method results and other values rises from the position of $0.001 \mathrm{~cm}$ to $10 \mathrm{~cm}$. It is obvious that present work with obtained GATE simulation in the lower energies is having more precisely calculation than other studies, because their values are identical until the $200 \mathrm{keV}$ energy. It is clearly seen in Figure 3 and Figure 4 that the total photon efficiency decreases with increasing the distance between the detector and the source. It causes most likely changing the solid angle by reducing the detector source distance, therefore, more photons interact with the scintillation. 

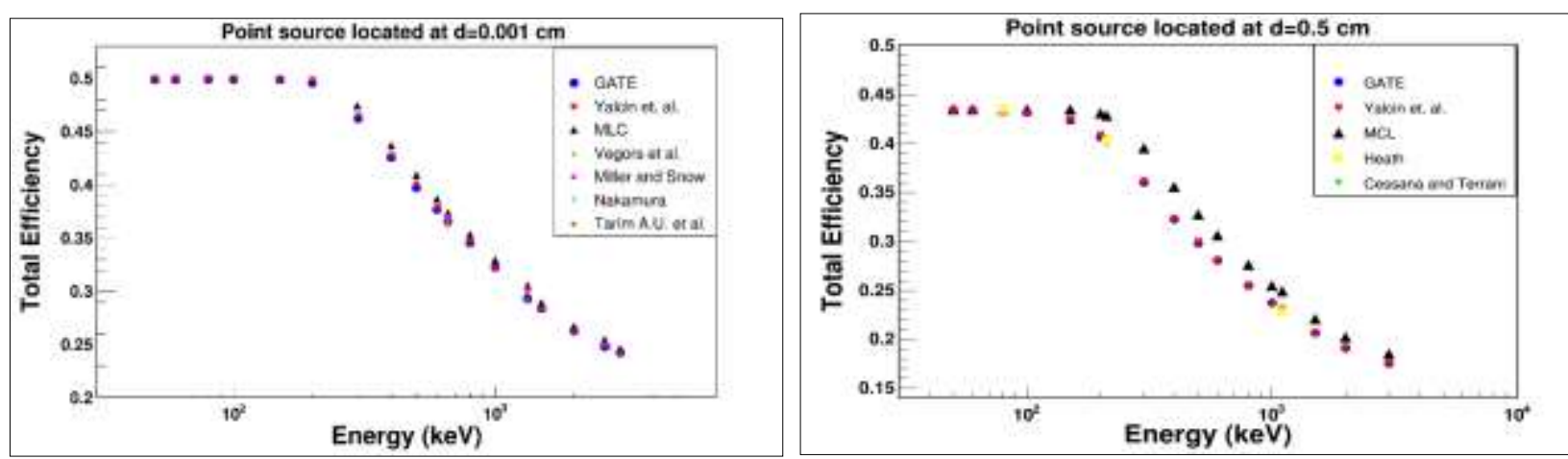

(a)

(b)

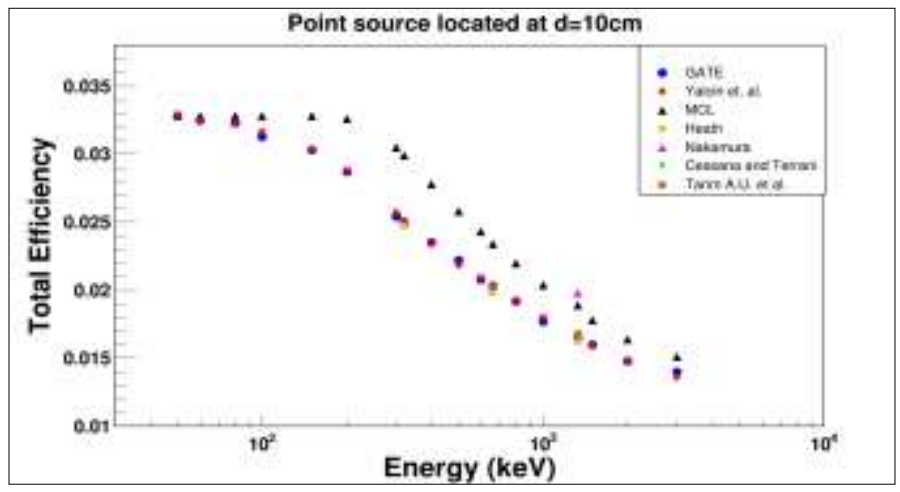

(c)

Figure 4. Total efficiency for a 3"x3" $\mathrm{NaI}(\mathrm{Tl})$ scintillator with a point source positioned at (a) $0.001 \mathrm{~cm}$ (b) $0.5 \mathrm{~cm}$ and (c) $10 \mathrm{~cm}$ distance between source and the detector surface

Table 1. Total counting efficiency values for a $3 " \mathrm{x} 3$ " NaI $(\mathrm{Tl})$ detector with disc source $(\mathrm{R}=3.81 \mathrm{~cm})$ located at $\mathrm{d}=3$ and $10 \mathrm{~cm}$

\begin{tabular}{|c|c|c|c|c|c|c|c|c|c|c|}
\hline \multirow{3}{*}{ 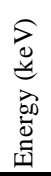 } & \multicolumn{10}{|c|}{ Total Efficiency } \\
\hline & \multicolumn{2}{|c|}{ Present Work } & \multicolumn{2}{|c|}{ Yalcin et al.[3] } & \multicolumn{2}{|c|}{$\begin{array}{c}\text { Cessana A. and } \\
\text { Terrani M.[24] }\end{array}$} & \multirow{2}{*}{\begin{tabular}{|c|} 
Nakamura T. \\
{$[25]$}
\end{tabular}} & \multirow{2}{*}{$\begin{array}{c}\begin{array}{c}\text { Belluscio } \\
\text { et al. [26] }\end{array} \\
10\end{array}$} & \multirow{2}{*}{$\begin{array}{c}\text { Heath R.L. } \\
{[28]} \\
3\end{array}$} & \multirow{2}{*}{$\begin{array}{c}\begin{array}{c}\text { Vegors } \\
\text { et al.[27] }\end{array} \\
10\end{array}$} \\
\hline & 3 & 10 & 3 & 10 & 3 & 10 & & & & \\
\hline 50 & 0.1462 & 0.0301 & 0.1466 & 0.0301 & - & - & - & - & - & - \\
\hline 60 & 0.1462 & 0.0299 & 0.1462 & 0.0299 & - & - & - & - & - & - \\
\hline 80 & - & 0.0299 & - & 0.0298 & - & - & - & - & - & - \\
\hline 81 & 0.1445 & - & 0.1448 & - & 0.143 & - & - & - & 0.145 & - \\
\hline 100 & 0.1429 & 0.0294 & 0.1428 & 0.0294 & - & - & - & - & - & - \\
\hline 150 & 0.1348 & 0.0284 & 0.1352 & 0.0282 & - & - & - & - & - & - \\
\hline 200 & 0.1255 & 0.0268 & 0.1257 & 0.0268 & - & - & - & - & - & - \\
\hline 212 & 0.1231 & - & 0.1236 & - & 0.123 & - & - & - & 0.123 & - \\
\hline 300 & 0.1084 & 0.024 & 0.1090 & 0.0241 & - & - & - & - & - & - \\
\hline 400 & 0.0971 & 0.0223 & 0.0979 & 0.0221 & - & - & - & - & - & - \\
\hline 500 & 0.0898 & 0.0207 & 0.0908 & 0.0208 & - & - & - & - & - & - \\
\hline 600 & 0.0857 & 0.0197 & 0.0855 & 0.0198 & - & - & - & - & - & - \\
\hline 661 & - & 0.0191 & - & 0.0191 & - & 0.0191 & 0.0183 & 0.019 & - & - \\
\hline 800 & 0.0784 & 0.0183 & 0.078 & 0.0182 & - & - & - & - & - & - \\
\hline 1000 & 0.0728 & 0.0172 & 0.0726 & 0.0171 & - & - & - & - & - & - \\
\hline 1100 & 0.0711 & - & 0.0711 & - & 0.0701 & - & - & - & 0.0702 & - \\
\hline 1332 & - & 0.0158 & - & 0.0159 & - & 0.0164 & 0.0168 & 0.0164 & - & 0.0156 \\
\hline 1500 & 0.0646 & 0.0152 & 0.0639 & 0.0151 & - & - & - & - & - & - \\
\hline 2000 & 0.0591 & 0.0143 & 0.0591 & 0.0141 & - & - & - & - & - & - \\
\hline 2620 & - & 0.0136 & - & 0.0135 & - & - & 0.0132 & - & - & 0.0133 \\
\hline 2750 & - & 0.0133 & - & 0.0134 & - & 0.0141 & - & 0.0141 & - & - \\
\hline 3000 & 0.0546 & 0.0133 & 0.0546 & 0.0131 & - & - & - & & - & - \\
\hline
\end{tabular}


Table 2. Total counting efficiency values for a $3 "$ x 3 " NaI(Tl) detector with point source located at d=0.001, 0.5 and $10 \mathrm{~cm}$

\begin{tabular}{|c|c|c|c|c|c|c|c|c|c|c|c|c|c|c|c|c|c|c|c|c|c|c|c|c|c|}
\hline & 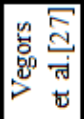 & 홍 & ' & ' & ' & & ' & ' & ' & & & 1 & ' & ' & $\begin{array}{l}\text { ర్ర } \\
\\
0\end{array}$ & ' & ' & ' & 1 & 1 & ふે & . & . & \begin{tabular}{l}
0 \\
\multirow{2}{*}{} \\
○े
\end{tabular} & . \\
\hline & 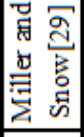 & $\ddot{8}$ & ' & ' & ' & & ' & ' & ' & & & 1 & ' & ' & 웅 & ' & ' & ' & 1 & 1 & ర్రి & 1 & 1 & 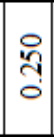 & ' \\
\hline & 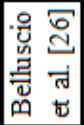 & 음 & ' & ' & ' & & ' & ' & ' & ' & ڤั) & ' & ' & ' & ' & $\begin{array}{l}2 \\
\\
\\
\end{array}$ & ' & ' & ' & \begin{tabular}{l}
\multirow{0}{0}{} \\
0 \\
0 \\
0
\end{tabular} & ' & 1 & 1 & 1 & ' \\
\hline & $\dot{A}_{\infty}$ & 으 & ' & ' & ' & & ' & ' & ' & ' & $\begin{array}{l}\text { 守 } \\
\text { ડุ } \\
\text { ¿ }\end{array}$ & ' & ' & ' & ' & $\begin{array}{c}\mathscr{2} \\
\stackrel{2}{\circ} \\
\circ \\
\end{array}$ & 1 & ' & ' & $\begin{array}{l}\widetilde{0} \\
\stackrel{-}{0} \\
0 \\
0\end{array}$ & , & 1 & 1 & ' & ' \\
\hline & 密 & ? & ' & 1 & $\begin{array}{c}\mathfrak{n} \\
\stackrel{v}{s} \\
\dot{c}\end{array}$ & & ' & ' & ô & ' & ' & ' & ' & ' & 1 & ' & ' & ' & તุ & & ' & ' & ' & ' & ' \\
\hline & 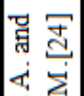 & 으 & ' & 1 & ' & & ' & ' & ' & ' & 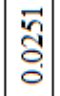 & ' & ' & ' & ' & 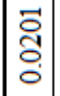 & ' & ' & ' & $\begin{array}{l}2 \\
\vdots \\
0 \\
0 \\
0\end{array}$ & ' & ' & ' & 1 & ' \\
\hline & 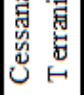 & ? & 1 & ' & 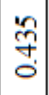 & ' & 1 & ' & $\begin{array}{l}\text { tे } \\
\text { के }\end{array}$ & ' & ' & ' & ' & ' & 1 & ' & ' & ' & તે & & 1 & ' & 1 & ' & ' \\
\hline & 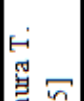 & 음 & ' & 1 & 1 & & ' & ' & 1 & 1 & ' & 1 & 1 & ' & ' & 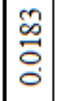 & . & 1 & ' & $\begin{array}{l}\infty \\
0 \\
\vdots \\
0 \\
0\end{array}$ & ' & ' & ' & ' & ' \\
\hline & 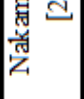 & 형 & ' & , & ' & & ' & ' & ' & & 1 & ' & ' & ' & $\begin{array}{l}\hat{0} \\
\\
0\end{array}$ & ' & . & ' & ' & ' & $\begin{array}{l}\text { ஓి } \\
\text { సి }\end{array}$ & ' & ' & 㝵 & ' \\
\hline $\begin{array}{l}\text { 节 } \\
\text { 密 } \\
\end{array}$ & 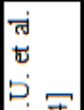 & 음 & & & & & & & & & & & & & 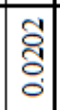 & & & & & & $\begin{array}{l}0 \\
0 \\
\circ \\
\circ \\
0\end{array}$ & & & & \\
\hline 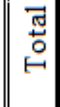 & 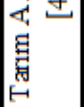 & 웅 & & & & & & & & & & & & & $\begin{array}{l}1 \\
6 \\
0 \\
0 \\
0\end{array}$ & & & & & & 영 & & & & \\
\hline & m & 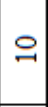 & ' & 1 & 1 & ' & ' & ' & & ' & 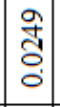 & ' & ' & ' & 1 & 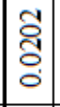 & , & ' & ' & \begin{tabular}{l}
\multirow{0}{0}{} \\
$\stackrel{0}{0}$ \\
$\stackrel{0}{0}$
\end{tabular} & , & 1 & ' & 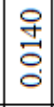 & ' \\
\hline & 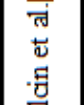 & "n & ' & ' & $\begin{array}{c}\text {m} \\
\text { m} \\
\stackrel{5}{\circ}\end{array}$ & & ' & ' & $\begin{array}{l}\stackrel{m}{0} \\
\stackrel{+}{+}\end{array}$ & & ' & ' & ' & ' & 1 & 1 & ' & 1 & ్ㅗㅇ & . & 1 & ' & ' & ' & ' \\
\hline & $\pi$ & 용 & ' & . & ' & & 1 & ' & ' & 1 & ' & 1 & ' & ' & 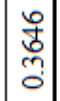 & ' & . & ' & ' & ' & 유 & , & , & 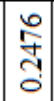 & , \\
\hline & 苞 & 으 & 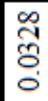 & $\begin{array}{l}0 \\
\text { בิ } \\
0 \\
0 \\
0\end{array}$ & 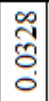 & 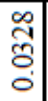 & 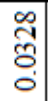 & $\begin{array}{l}\text { ते } \\
\text { ชิ } \\
0\end{array}$ & , & 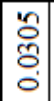 & 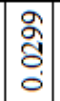 & 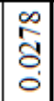 & 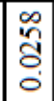 & 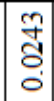 & ' & 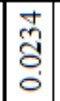 & ป⿱宀 & 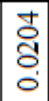 & ' & $\begin{array}{l}\text { के } \\
\stackrel{0}{-} \\
0 \\
0\end{array}$ & ' & 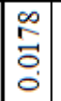 & $\begin{array}{l} \\
0 \\
0 \\
0 \\
0 \\
0\end{array}$ & ' & 름 \\
\hline & 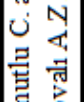 & ? & 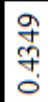 & 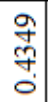 & 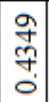 & 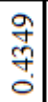 & 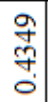 & 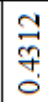 & 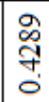 & 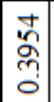 & , & 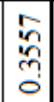 & Ê & 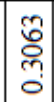 & . & , & 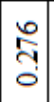 & ปั & 竎 & , & ' & 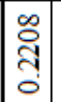 & กิ &. & 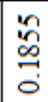 \\
\hline & $0 \stackrel{N}{5}$ & 홍 & 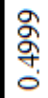 & 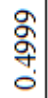 & 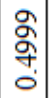 & 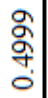 & 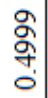 & $\begin{array}{l}+ \\
\text { o } \\
\text { +े } \\
0\end{array}$ & , & 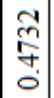 & 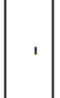 & 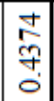 & 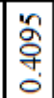 & $\begin{array}{c}n \\
\infty \\
\tilde{0} \\
0\end{array}$ & 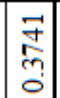 & , & 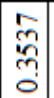 & 㐫 & ' & ' & 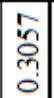 & 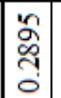 & $\overrightarrow{\widehat{\sigma}}$ & นู & 吉 \\
\hline & & 으 & 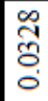 & 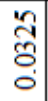 & 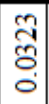 & $\begin{array}{l}9 \\
\stackrel{9}{2} \\
\stackrel{8}{0} \\
0\end{array}$ & \begin{tabular}{l|}
3 \\
0 \\
0 \\
0 \\
0 \\
0
\end{tabular} & 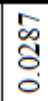 & , & 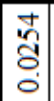 & $\begin{array}{l}9 \\
\text { ปे } \\
\text { ○े } \\
0\end{array}$ & 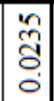 & 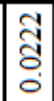 & 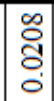 & 1 & 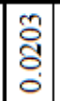 & \begin{tabular}{|l|}
\multirow{2}{\alpha}{} \\
$\vdots$ \\
0 \\
0
\end{tabular} & 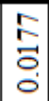 & ' & $\begin{array}{l}0 \\
0 \\
0 \\
0 \\
0\end{array}$ & ' & $\begin{array}{l}0 \\
0 \\
0 \\
0 \\
0 \\
0\end{array}$ & \begin{tabular}{|l|} 
\\
\\
\\
\\
0 \\
\end{tabular} & ' & $\begin{array}{l}\text { 움 } \\
\text { 웅 } \\
\end{array}$ \\
\hline & 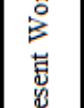 & ? & 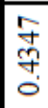 & 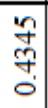 & $\begin{array}{l}\mathrm{n} \\
\mathfrak{m} \\
\tilde{c} \\
0\end{array}$ & 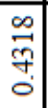 & 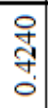 & $\begin{array}{l}0 \\
0 \\
\vdots \\
\vdots \\
0\end{array}$ & 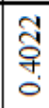 & \begin{tabular}{|c|}
$\overrightarrow{0}$ \\
0 \\
0 \\
0 \\
\end{tabular} & ' & 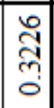 & 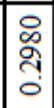 & 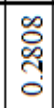 & . & , & 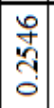 & 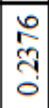 & ปิ & , & . & 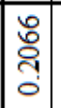 & 음 & & 告 \\
\hline & $\vec{m}$ & 훙 & 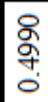 & 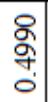 & 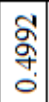 & 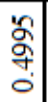 & $\begin{array}{l}\bar{g} \\
\text { aे } \\
\text { ○े }\end{array}$ & 今. & . & 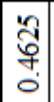 & , & 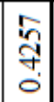 & $\mid \begin{array}{l}n \\
\hat{a} \\
\tilde{o} \\
0\end{array}$ & 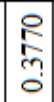 & $\begin{array}{c}8 \\
0 \\
0 \\
0 \\
0 \\
0\end{array}$ & , & 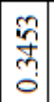 & ปิ & . & . & ふે̀ & 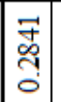 & 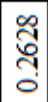 & 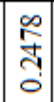 & 志 \\
\hline & 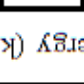 & & 으 & 8 & ৪ & 8 & 吕 & ষ্ণ & 걱 & \begin{tabular}{|l|} 
\\
\end{tabular} & శి & \begin{tabular}{|l|} 
\\
\end{tabular} & 8 & 8 & $\vec{b}$ & तु & ৪) & 8 & $\stackrel{8}{\circ}$ & $\begin{array}{l}\text { O } \\
\text { m. } \\
\end{array}$ & \begin{tabular}{|c|} 
In \\
-
\end{tabular} & \begin{tabular}{|l|}
8 \\
$n$ \\
\end{tabular} & $\begin{array}{l}8 \\
\text { ¿े }\end{array}$ & \begin{tabular}{|l|} 
ర్రి \\
\end{tabular} & ষ্ঠ \\
\hline
\end{tabular}


In figure 5 there is a comparison between recently published efficiency values obtained from a simple computational Monte Carlo algorithm (developed by Tarım et. al.) and Geant4 based GATE simulation results. In this part of the simulation 2"x2" cylindrical $\mathrm{NaI}(\mathrm{Tl})$ detector system was defined and three different source-detector distance was used $(0.001,5$ and $10 \mathrm{~cm})$. It is clearly seen that the calculated and simulated values of the efficiencies for $\mathrm{NaI}(\mathrm{Tl})$ detector indicated good agreement.

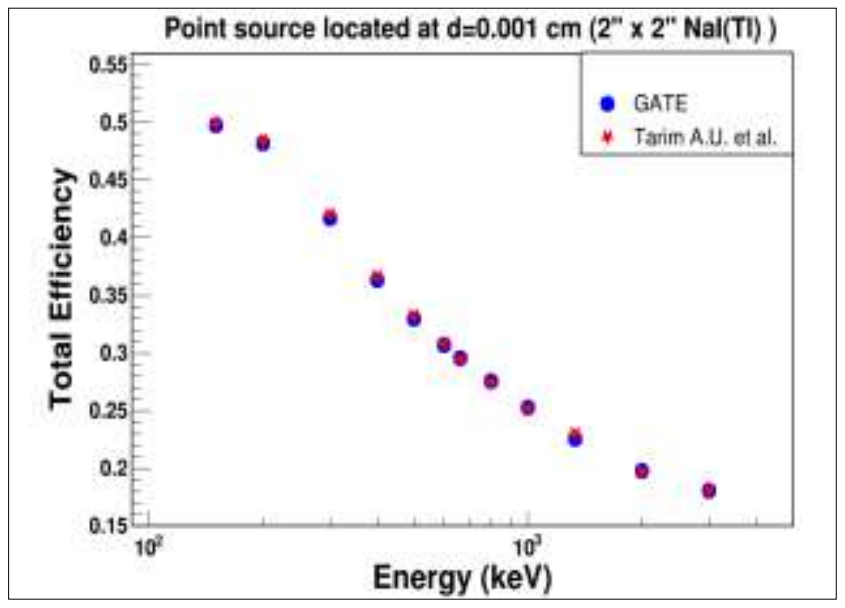

(a)

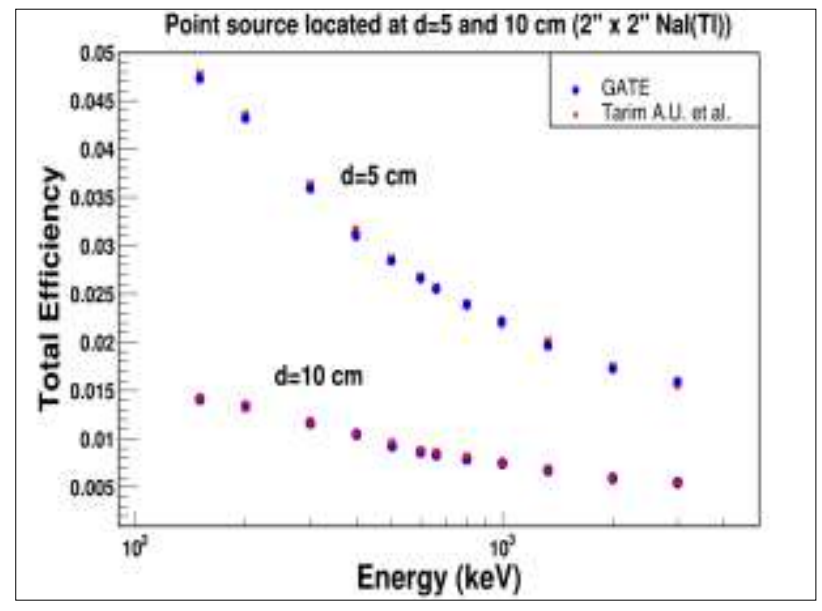

(b)

Figure 5. Total efficiency for a $2 " \mathrm{x} 2 " \mathrm{NaI}(\mathrm{Tl})$ scintillation detector with a point source positioned at (a) 0.001 $\mathrm{cm}$ (b) $5 \mathrm{~cm}$ and $10 \mathrm{~cm}$ distance between source and the detector surface

\section{Conclusion}

In this study, the total efficiency of the cylindrical NaI(Tl) (3" x 3") and (2" x 2") detectors have been calculated for isotropic point and disc gamma-rays source by using Geant 4 based GATE simulation program. To compare our results with other different analytical and Monte Carlo calculation results we have evaluated the efficiencies in the photon energy range 50-3000 keV and for $0.001 \mathrm{~cm}, 3 \mathrm{~cm}, 5 \mathrm{~cm}$ and $10 \mathrm{~cm}$ distance between the source and the detector. The results are shown in table 1, table 2 and figures the agreement is quite good in the whole energy range. However, we can say that Geant 4 gives more sensitive results in the low energetic photon (between 50 and $200 \mathrm{keV}$ ).

\section{References}

[1] Zazubovich S. 2001. Physics of halide scintillators. Radiation Measurements, 33 (5): 699-704.

[2] Mouhtia I., Elaniquea A., Messousb M.Y., Benahmedb A., McFeec J.E., Elgouba Y., Griffithd P. 2019. Characterization of $\mathrm{CsI}(\mathrm{Tl})$ and $\operatorname{LYSO}(\mathrm{Ce})$ scintillator detectors by measurements and Monte Carlo simulations. Applied Radiation and Isotopes, 154 (108878): 1-7.

[3] Yalcin S., Gurler O., Kaynak G., Gundogdu O. 2007. Calculation of total counting efficiency of a $\mathrm{NaI}(\mathrm{Tl})$ detector by hybrid Monte-Carlo method for point and disc sources. Applied Radiation and Isotopes, 65: 1179-1186.

[4] Tarım Akar U., Gürler O., Yalçın S. 2018. A Quick Method to Calculate NaI(Tl) Detector Efficiency Depending on Gamma ray Energy and Source-to-detector Distance. Celal Bayar University Journal of Science, 14 (2): 195-199.

[5] Hofstadter R. 1949. The Detection of Gamma-Rays with Thallium-Activated Sodium Iodide Crystals. Physical Review, 75 (5): 796-810.

[6] Pilakouta M., Pappa F.K., Patiris D.L., Tsabaris C., Kalfas C.A. 2018. A methodology for expanding the use of $\mathrm{NaI}(\mathrm{Tl})$ based spectrometry in environmental radioactivity measurements. Applied Radiation and Isotopes, 139: 159-168. 
[7] Zhang Y., Li C., Liu D., Zhang Y., Liu Y., 2015. Monte Carlo simulation of a NaI(Tl) detector for in situ radioactivity measurements in the marine environment. Applied Radiation and Isotopes, 98: 44-48.

[8] Duc Tam H., Hai Yen N.T., Tran, L.B., Dinh Chuong, H., Thien Thanh, T. 2017. Optimization of the Monte Carlo simulation model of $\mathrm{NaI}(\mathrm{Tl})$ detector by Geant 4 code. Applied Radiation and Isotopes, 130: 75-79.

[9] Wang J., Zhang Y., Liu D., Wu B., Zhang Y., Jiang H., 2018. Automated spectra analysis of in situ radioactivity measurements in the marine environment using $\mathrm{NaI}(\mathrm{Tl})$ detector. Applied Radiation and Isotopes, 141: 88-94.

[10] Hamzawy A., 2010. Simple analytical formula to calculate $\gamma$-ray cylindrical detectors efficiencies. Nuclear Instruments and Methods in Physics Research Section A, 624 (1): 124-129.

[11] Chuong H.D., Nguyen Q.H., Nguyen T.M.L., Nguyen V.H. 2019. Validation of gamma scanning method for optimizing $\mathrm{NaI}(\mathrm{Tl})$ detector model in Monte Carlo simulation. Applied Radiation and Isotopes, 149: 1-8

[12] Zikovsky L., Chah B. 1988. A computer program for calculating Ge(li) detector counting efficiencies with large volume samples. Nuclear Instruments and Methods in Physics Research, A263 (2-3): 483-486.

[13] Özmutlu C., Ortaovalı A.Z. 1976. Calculation of total and full energy peak efficiencies of Ge(Li) and $\mathrm{NaI}(\mathrm{Tl})$ detectors by introducing the mean chord length. Nuclear Instruments and Methods, 149-155.

[14] Selim Y.S., Abbas M.I., Fawzy M.A. 1998. Analytical calculation of the efficiencies of gamma scintillators. Part I: Total efficiency for coaxial disk sources. Radiation Physics and Chemistry, 53: 589-592.

[15] Abbas M.I. 2010, A new analytical method to calibrate cylindrical phoswich and $\mathrm{LaBr} 3(\mathrm{Ce})$ scintillation detectors. Nuclear Instruments and Methods in Physics Research A, 621: 413-418.

[16] Abbas M.I., Noureddeen S. 2011. Analytical expression to calculate total and full-energy peak efficiencies for cylindrical phoswich and lanthanum bromide scintillation detectors. Radiation Measurement, 46: 440-445

[17] Pomme S. 2009. Detection efficiency calculation for photons, electrons and positrons in a well detector. Part I: Analytical model. Nuclear Instruments and Methods in Physics Research A, 604: 584-591.

[18] Lépy M.C. et al. 2019. A benchmark for Monte Carlo simulation in gamma-ray spectrometry. Applied Radiation and Isotopes, 154 (108850): 1-7

[19] Ashrafi S., Anvarian S., Sobhanian S. 2006. Monte-Carlo modeling of a NaI(Tl) scintillator. Journal of Radioanalytical and Nuclear Chemistry, 269 (1): 95-98.

[20] Garnett R., Prestwich W.V., Atanackovic J., Wong M., Byun S.H. 2017. Characterization of a $\mathrm{LaBr} 3(\mathrm{Ce})$ detector for gamma-ray spectrometry for CANDU power reactors. Radiation Measurements, 106: 628-631.

[21] Zhang J., Zhang P., Zhang Y., Yang J., Yuan G., Song X., Li X., Zhou Y. 2019. Geant4 simulation study on detection efficiencies of the Compton suppression system at the HL-2A tokamak. Applied Radiation and Isotopes, 150: 63-69.

[22] Wirawan R., Angraini L.M., Qomariyah N., Waris A., Djamal M. 2020. Gamma backscattering analysis of flaw types and orientation based on Monte Carlo Geant4 simulations. Applied Radiation and Isotopes, 155 (108924): 1-7.

[23] Open Gate Collaboration "Users Guide V8.0 From Wiki OpenGATE". 2018. http://www.opengatecollaboration.org/sites/default/files/GATE-UsersGuideV8.0.pdf (Accessed July 2018).

[24] Cesana A., Terrani M. 1977. Gamma-ray Activity Determination in Large Volume Samples with a Ge-Li Detector. Analytical Chemistry, 49 (8): 1156-1159.

[25] Nakamura T. 1972. Monte Carlo calculation of efficiencies and response functions of $\mathrm{NaI}(\mathrm{Tl})$ crystals for thick disk gam-ray sources and its application to $\mathrm{Ge}(\mathrm{Li})$ detectors. Nuclear Instruments and Methods, 105: 77-89. 
[26] Belluscio M., DeLeo R., Pantaleo A., Vox A. 1974. Efficiencies and response functions of $\mathrm{NaI}(\mathrm{T} 1)$ crystals for gamma rays from thick disk sources. Nuclear Instruments and Methods, 118: 553-563.

[27] Vegors Jr, S.H, Marsden, L.L, Heath, R.L. 1958. Calculated efficiencies of cylindrical radiation detectors. AEC Research and Development Report, 1958, IDO-16370.

[28] Heath R.L. 1964. Scintillation spectrometry. Vol. 1, IDO-16880-1.

[29] Miller W.F., Snow W.J., 1961. NaI and CsI efficiencies and photofractions for gamma-ray detection. Nucleonics, 19 (11): 174.

[30] ROOT Date Analysis Framework user's guide. 2018. https://root.cern.ch/root/htmldoc/guides/users-guide/ROOTUsersGuide.html (Accessed September 2018). 